\title{
Correction: Comparison of individually tailored versus fixed- schedule rituximab regimen to maintain ANCA-associated vasculitis remission: results of a multicentre, randomised controlled, phase III trial (MAINRITSAN2)
}

Charles P, Terrier B, Perrodeau É, et al. Comparison of individually tailored versus fixedschedule rituximab regimen to maintain ANCA-associated vasculitis remission: results of a multicentre, randomised controlled, phase III trial (MAINRITSAN2). Ann of Rheum Dis 2018;77:1143-9.

Table 1 has been corrected. The figures concerning ANCA-positivity rates at inclusion advertently inversed.

\begin{tabular}{|c|c|c|}
\hline \multirow[b]{2}{*}{ Characteristic } & \multicolumn{2}{|l|}{ Rituximab infusions } \\
\hline & Individually tailored $(\mathrm{N}=81$ ) & Fixed-schedule $(\mathrm{N}=81)$ \\
\hline Age $-y r$ & $62 \pm 14$ & $59 \pm 13$ \\
\hline Female sex — no. (\%) & $31(38.3)$ & $37(45.7)$ \\
\hline \multicolumn{3}{|l|}{ Vasculitis type - no. (\%) } \\
\hline GPA & $56(69.1)$ & $61(75.3)$ \\
\hline MPA & $25(30.9)$ & $20(24.7)$ \\
\hline \multicolumn{3}{|l|}{ Disease status — no. (\%) } \\
\hline Newly diagnosed & $53(65.4)$ & $51(63.0)$ \\
\hline Relapsing & $28(34.6)$ & $30(37.0)$ \\
\hline \multicolumn{3}{|c|}{ Induction treatment of last disease flare - no. (\%) } \\
\hline Cyclophosphamide & $52(64.2)$ & $49(60.5)$ \\
\hline RTX & $28(34.6)$ & $32(39.5)$ \\
\hline Methotrexate & $1(1.2)$ & $0(0.0)$ \\
\hline Prednisone dose $(\mathrm{mg})$; median (IQR) & $10(10-15)$ & $12(10-17.3)$ \\
\hline \multicolumn{3}{|l|}{ Organ involvement at last flare — no. (\%) } \\
\hline Ear, nose and throat & $46(56.8)$ & $39(48.1)$ \\
\hline Pulmonary & $50(61.7)$ & $44(54.3)$ \\
\hline Renal & $60(74.1)$ & $56(69.1)$ \\
\hline $\mathrm{GFR}-\mathrm{ml} / \mathrm{min} / 1.73 \mathrm{~m}^{2}$ at inclusion & $55.6 \pm 27.3$ & $58.9 \pm 27.0$ \\
\hline ANCA-positive at diagnosis - no. $(\%)^{*}$ & $74 / 77(96.1)$ & $72 / 79(91.1)$ \\
\hline Indirect immunofluorescence & $68 / 77(88.3)$ & $6579(82.3)$ \\
\hline ELISA & $64 / 77(83.1)$ & $61 / 79(77.2)$ \\
\hline Anti-PR3 & $38 / 77(49.4)$ & $38 / 79(48.1)$ \\
\hline Anti-MPO & $26 / 77(33.8)$ & $24 / 79(30.4)$ \\
\hline ANCA-positive at inclusion — no. $(\%) \dagger$ & $58 / 80(72.5)$ & $45 / 80(56.3)$ \\
\hline Indirect immunofluorescence & $54 / 80(67.5)$ & $40 / 80(50)$ \\
\hline ELISA & $43(53.7)$ & $28(35)$ \\
\hline Anti-PR3 & $21(26.2)$ & $18(22.5)$ \\
\hline Anti-MPO & $23(28.7)$ & $10(12.5)$ \\
\hline
\end{tabular}

Plus-minus values are means \pm SD. GPA denotes granulomatosis with polyangiitis, MPA microscopic polyangiitis, ANCA antineutrophil cytoplasm antibodies, PR3 proteinase-3, MPO myeloperoxidase and GFR glomerular filtration rate. *Data were missing for 4 individually tailored-infusion and 2 fixed-schedule patients. tData were missing for 1 patient in each group.

Also, the sentence in the Limitation section of the Discussion "Fixed-schedule infusion-arm patients were more likely to be ANCA-positive at inclusion." should read "Fixed-schedule infusion-arm patients were less likely to be ANCA-positive at inclusion."

(c) Author(s) (or their employer(s)) 2019. No commercial re-use. See rights and permissions. Published by BMJ. Ann Rheum Dis 2019;78:e101. doi:10.1136/annrheumdis-2017-212878corr1

Check for updates 